\title{
Exercise duration during pulmonary rehabilitation: an index of efficacy
}

\author{
Hoda Mojazi Amiri, MD, Ryan McClellan, BS, Chok Limsuwat, MD, Kenneth Nugent, MD
}

\begin{abstract}
Background: The optimal program for pulmonary rehabilitation likely varies from patient to patient and depends on individual limiting factors. We wanted to determine the typical exercise duration in our patients to use as a basis for modifying individual rehabilitation components.

Methods: We retrospectively reviewed the medical records of patients who completed the pulmonary rehabilitation program at University Medical Center in Lubbock, TX. We collected demographic, pulmonary function, and 6-minute walk test information. Patients exercised with treadmills, arm ergometers, recumbent stair steppers, and recumbent bicycles; we recorded the initial and follow-up modality duration at four week intervals.

Results: This study included 119 patients with a mean age of $68.8 \pm 10.1$ years. Most patients (95) had COPD. The mean FEV1 was $1.3 \pm 0.7$ L 9 (47.2 $\pm 19.7 \%$ predicted). The baseline gait speed was $41 \pm 15$ meters per minute. The initial total exercise duration was $30.5 \pm 7.0$ minutes. These times increased to $41.2 \pm 8.8,44.6 \pm 10.7$ and $47.0 \pm 12.0$ minutes at 4, 8 and 12 weeks of rehabilitation.

Conclusions: Most patients increased their total exercise duration to 40 minutes. This goal can identify patients who are not making progress and need alternative strategies and patients who have made good progress and might benefit from additional training, such as lower extremity strength exercises.
\end{abstract}

Key words: exercise, endurance, chronic lung disease, pulmonary rehabilitation, modality

Pulmonary rehabilitation programs provide significant benefit to patients with chronic lung disease; these include increased physical endurance, less dyspnea, improved health-related quality of life, and possibly decreased health care costs ${ }^{1}$. Comprehen-

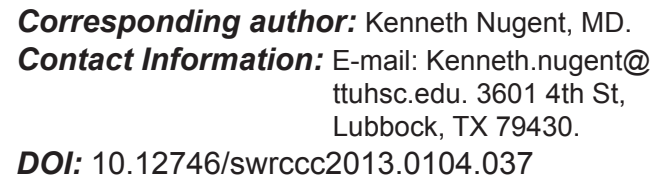

DOI: $10.12746 /$ swrccc2013.0104.037

sive multicomponent sessions likely provide the best outcomes. However, the best strategy and use of time probably varies from patient to patient ${ }^{2-4}$. Our program emphasizes aerobic fitness using four exercise modalities, including the treadmill, a recumbent bicycle, an arm ergometer, and a recumbent stair stepper. Individual adjustments to the exercise prescription are made as needed at each session based on recent progress, vital signs, and any ongoing health problems. We wanted to determine the usual exercise duration in our patients over the course of a rehabilitation program to establish a basis for modifying rehabilitation in individual patients. 


\section{METHODS}

Subjects: We retrospectively collected information on patients who completed pulmonary rehabilitation at University Medical Center (Lubbock, TX) in the years 2010, 2011, and the first half of 2012. These patients have been referred to the rehabilitation center by their private physicians and met Medicare criteria for eligibility for pulmonary rehabilitation. We included all patients who completed at least two 6-minute walk tests. We did not limit patient entry to particular diagnoses. We collected information of demographics, medical diagnoses, current symptoms, medication use, pulmonary function results, and questionnaire information, including SF36 and a dyspnea score. The dyspnea index was taken from the "Guidelines for Pulmonary Rehabilitation" 2nd edition, 1998, chapter 2, page 24. There are 14 questions about dyspnea in routine situations ranging from "at rest" to "walking up a hill" with responses ranging from 1 (minimal) to 4 (unable to do). We collected information on comorbidities using the Charlson index. Patients completed 6-minute walk tests at the beginning of the rehabilitation program, every 4 weeks during the program, and at completion of rehabilitation. The distance was recorded in feet, and the gait speed was calculated using the time and the distance after conversion into meters and reported as meters per minute. This study was approved by the Institutional Review Board at Texas Tech University Health Sciences Center in Lubbock, Texas.

Rehabilitation protocol: During each rehabilitation session, vital signs and modality durations were recorded. Patients exercised on treadmills, recumbent bicycles, arm ergometers, and recumbent stair steppers; the program was individualized to obtain optimal performance and endurance. The goal was to increase modality duration to 15 minutes per modality per session. Individual adjustments to the exercise prescription were made at each session based on recent progress, current vital signs, changes in symptoms which might represent early flares, and recent medical care.

Analysis: Descriptive information was analyzed using means \pm standard deviations, medians with interquartile ranges, and counts (frequency) and percentages. Comparisons between groups were made with t-tests, paired t-tests, or chi-square tests. Differences in machine time over the rehabilitation program were compared using analysis of variance. These data were analyzed with SPSS version 20 (IBM, Inc, Armonk, NY), and P-values $\leq 0.05$ were considered statistically significant.

\section{RESULTS}

This study included 119 patients with a mean age of $68.8 \pm 10.1$ years. Sixty-two subjects were men $(52.1 \%)$, and most patients had COPD/asthma (95, $79.8 \%)$. Other diagnoses included pulmonary fibrosis $(14,11.8 \%)$, obstructive sleep apnea $(7,5.9 \%)$, and miscellaneous disorders (3, $2.5 \%)$. The mean comorbidity index was $0.92 \pm 1.0$. Ninety-seven percent of subjects reported significant dyspnea with a median score of 24 (interquartile $25 \%$ to $75 \%$ range: $16-33$ ). The initial 6-minute walk distance was $215.5 \pm 110.7$ meters; the final distance was $260.7 \pm 109.1$ meters. The initial gait speed was $41 \pm 15$ meters per minute; the final gait speed was $47 \pm 15$ meters per minute. The overall program duration in rehabilitation was $19.3 \pm 7.4$ weeks; this time included gaps for acute illness and unavoidable personal and family responsibilities.

The mean baseline total modality duration was $30.5 \pm 7.0$ minutes; the median was 30.0 minutes (interquartile range: 26-34 minutes).The distribution of initial times is displayed in Figure 1. Total modality time increased after four weeks and appeared to plateau at four weeks (Table 1). The modality durations at 4,8 , and 12 weeks were significantly higher than the baseline time ( $p<0.05$ by analysis of variance). There were significant correlations between the final total modality time at the end of rehabilitation and both the 6-minute walk distance after rehabilitation $(r=0.313, P=0.001)$ and the change in 6-minute walk distance after rehabilitation $(r=0.251, P=0.006)$. Calorie consumption on the treadmill at base line was $22.6 \pm 11.2$ calories (based on METS and time); this increased to $42.4 \pm 22.2$ calories at 12 weeks. 


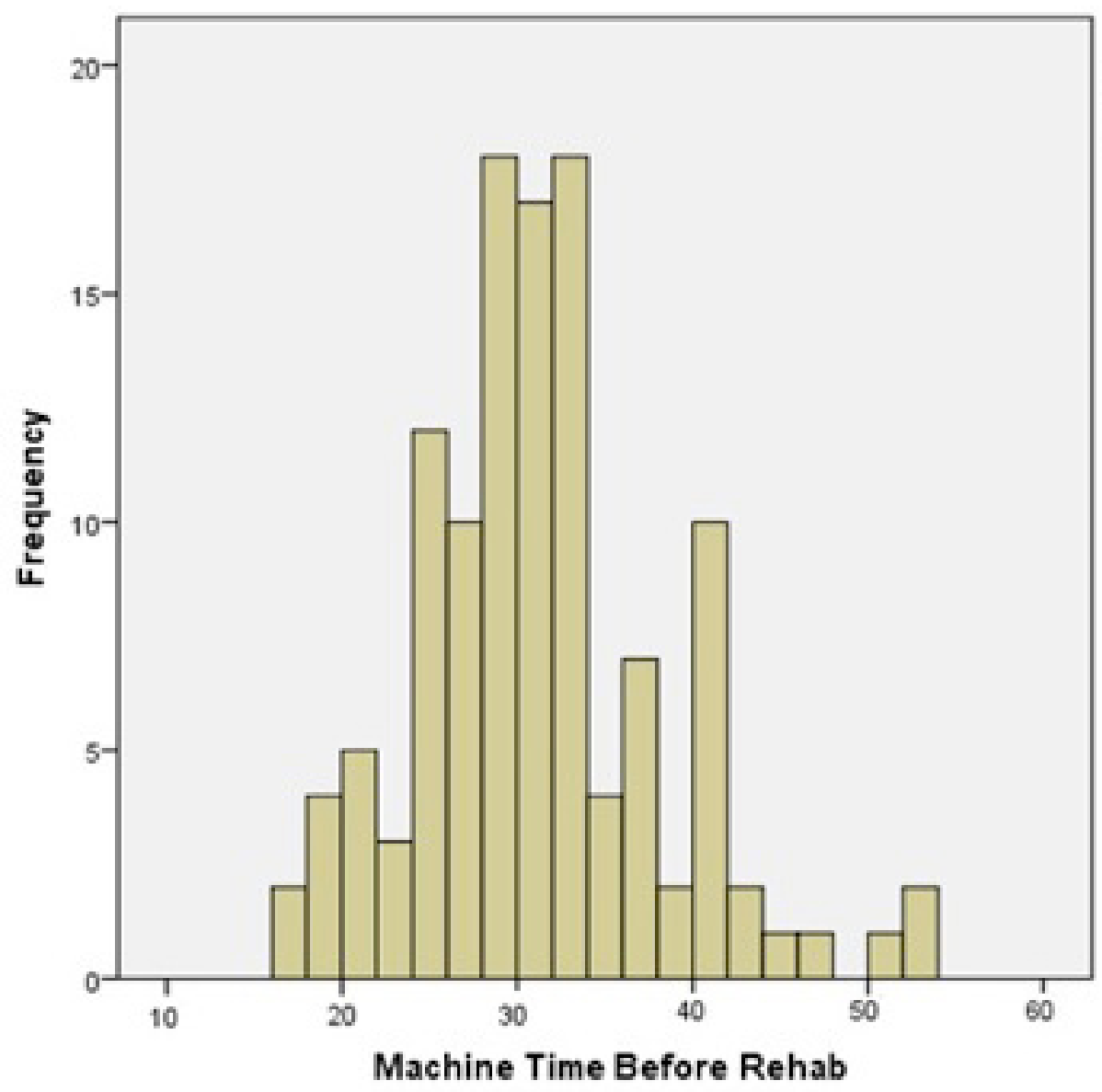

Baseline exercise duration for the entire cohort

Table 1: Exercise time over the course of pulmonary rehabilitation

\begin{tabular}{|l|c|c|c|c|l|}
\hline $\begin{array}{l}\text { Time inter- } \\
\text { val }\end{array}$ & Treadmill & Stair Stepper & $\begin{array}{l}\text { Arm Ergom- } \\
\text { eter }\end{array}$ & $\begin{array}{l}\text { Recumbent } \\
\text { Bicycle }\end{array}$ & Total Time \\
\hline Baseline & $7.9 \pm 2.7^{*}$ & $8.4 \pm 2.2$ & $7.8 \pm 1.7$ & $7.7 \pm 1.6$ & $30.5 \pm 7.0$ \\
\hline Week 4 & $10.7 \pm 4.1$ & $11.2 \pm 2.6$ & $10.6 \pm 2.5$ & $10.4 \pm 2.2$ & $41.2 \pm 8.8$ \\
\hline Week 8 & $12.0 \pm 4.4$ & $12.3 \pm 3.1$ & $11.9 \pm 2.9$ & $11.4 \pm 2.8$ & $44.6 \pm 10.7$ \\
\hline Week 12 & $12.7 \pm 4.8$ & $13.3 \pm 3.6$ & $12.6 \pm 3.2$ & $12.5 \pm 3.1$ & $47.0 \pm 12.0$ \\
\hline & & & & & \\
\hline
\end{tabular}

${ }^{*}$ Time in minutes 


\section{Discussion}

Our patients increased their total exercise times on the four machines used to improve aerobic fitness, their 6-minute walk distances, and their gait speeds during rehabilitation. Our results suggest that we should expect patients to increase their total machine time to 40 minutes after four weeks of participation. Patients below this level need to be reevaluated to consider limiting factors and possible changes in their program. Patients above 40 minutes should have their work load increased during exercise and spend additional time with other activities, such as upper and lower extremity strength training.

Outcomes assessments with pulmonary rehabilitation programs can be based on sophisticated measurements, such VO2 max and maximum watts using incremental exercise tests, endurance time on an exercise cycle, changes in daily activity, 6-minute walk distances, and, perhaps, patient specific goals. Modality time provides a relatively simple measure of progress and correlates with six minute walk distance in our patient cohort. However, it may not correlate with VO2 max. Ong and coworkers reported that changes in VO2 max correlated with changes in maximum watts but not with other outcome parameters ${ }^{6}$. Therefore, improvement in one physical activity may not correlate with changes in another. The importance of this will depend on the patient and program goals. High intensity training may help patients achieve new physical goals, whereas low intensity training may largely help patients sustain current activities with less discomfort ${ }^{6}$. Also, the training method and requisite assessment will affect equipment and testing requirements and costs.

High intensity training aims for workloads at $80 \%$ of VO2 max and above the anaerobic threshold. Training sessions will need heart rate monitoring and close supervision. Low intensity training involves sustained exercise at lower VO2s and can be based on time and symptoms. Interval training alternates from high intensity to low intensity and is based on time and symptoms. Most studies suggest that outcomes with high intensity training are similar to lower intensity continuous training ${ }^{7-11}$. Gloeckl and coworkers compared interval training (30 seconds at peak work followed by 30 seconds of rest) with continuous training at $60 \%$ of peak work in 60 lung transplant candidates ${ }^{8}$. The 6-minute walk distance increased 35 meters in both groups. Many older chronically ill patients will find high intensity session very uncomfortable. In addition, this approach likely requires more personnel time to monitor safety and provide motivation. We base our training sessions on symptoms and had a similar increase in 6-minute walk distance. This approach eliminates the need for formal exercise testing and possibly increases patient comfort during rehabilitation sessions.

Rehabilitation programs need to balance individual patients' goals with available time. In our opinion the program goal for most patients should include: treadmill (15 minutes), arm ergometer (10 minutes), stair stepper (10 minutes), and bicycle (10 minutes). These goals focus on lower extremity strength and endurance which are crucial for routine daily activities. As the patient builds up aerobic conditioning, strength training should be added with a focus on the legs. Since time constraints limit the total time available for supervised exercise, patients should walk at least 20 minutes and use light arm weights on off days ${ }^{12,13}$. Patients need frequent monitoring during this process, and gait speed measurements provide a quick assessment of progress. Patients who do not reach 40 minutes of total exercise time by four weeks need reassessment to identify all limiting factors.

\footnotetext{
TTUHSC.

Received: 9/16/2013

Accepted: 10/16/2013

Reviewers: Dean Diersing MS, Rishi Raj MD

Published electronically: 10/15/2013

Conflict of Interest Disclosures: None
}

Author Affiliation: Hoda Mojazi Amiri is a resident in Internal medicine at the Eisenhower Medical Center in Rancho Mirage, CA; Ryan McClellan is a medical student in the SOM at TTUHSC; Chok Limsuwat is a sleep medicine fellow at the Tulane University in New Orleans, LA; and Kenneth Nugent is a pulmonary medicine physician at 


\section{REFERENCES}

1. Goldstein RS, Hill K, Brooks, D, Dolmage TD. Pulmonary rehabilitation: a review of the recent literature. Chest 2012; 142:738-749.

2. Pepin V, Saey D, Laviolette L, Maltais F. Exercise capacity in chronic obstructive pulmonary disease: mechanisms of limitation. COPD: Journal of Chronic Obstructive Pulmonary Disease 2007; 4:195-204.

3. Vivodtzev I, Gagnon P, Pepin V, et al. Physiological correlates of endurance time variability during constant-work rate cycling exercise in patients with COPD. Plos One 2011; 6:

4. Plankeel JF, McMullen B, MacIntyre NR. Exercise outcomes after pulmonary rehabilitation depend on the initial mechanism of exercise limitation among non-Oxygen-dependent COPD patients. Chest 2005; 127:110-116.

5. Ong KC, Chong WF, Soh C, Earnest A. Comparison of different exercise tests in assessing outcomes of pulmonary rehabilitation. Respir Care 2004; 49:1498-503.

6. Butcher SJ, Jones RL. The impact of exercise training intensity on change in physiological function in patients with chronic obstructive pulmonary disease. Sports Med 2006; 36:307-25.

7. Kortianou EA, Nasis IG, Spetsioti ST, MSc, Daskalakis AM, Vogiatzis I. Effectiveness of interval exercise training in patients with COPD. Cardiopulm Phys Thera J 2010; 21:12-19.

8. Gloeckl R, Halle M, Kenn K. Interval versus continuous training in lung transplant candidates: A randomized trial. J Heart Lung Transpl 2012; 31:934-941.

9. Beauchamp MD, Nonoyama M, Goldstein RS, et al. Interval and continuous training are similarly effective on chronic obstructive pulmonary disease. Thorax, 2010; 65:157-64.

10. Hsieh MJ, Lan CC, Chen NH, Huang CC, Wu YK, Cho HY, Tsai YH. Effects of high- intensity exercise training in a pulmonary rehabilitation programme for patients with chronic obstructive pulmonary disease. Respirology 2007; 12:381-8.

11. Arnardottir RH, Boman G, Larsson $\mathrm{K}$, Hedenstrom $H$, Emtner M. Interval training compared with continuous training in patients with COPD. Resp Med 2007, 101:1196-1204. 12. Ferrari M, Vangelista A, Vedovi E, Falso M, Segattini C, Brotto E, Brigo B, Lo Cascio V. Minimally supervised home rehabilitation improves exercise capacity and health status in patients with COPD. Am J Phys Med Rehabil 2004; 83:337-43.

13. Pomidori L, Contoli M, Mandolesi G, Cogo A. A simple method for home exercise training in patients with chronic obstructive pulmonary disease. J Cardiopulm Rehab Prev 2012; 32:53-57. 